\title{
LA TEORÍA DISTRIBUTIVA DE DWORKIN Y EL DERECHO A LA PROTECCIÓN DE LA SALUD*
}

\section{AlejandRa ZÚNíga FAJURI**}

RESUMEN: En este artículo se pasa revista a la teoría de distribución sanitaria del filósofo estadounidense Ronald Dworkin, la que se desarrolla bajo la premisa de la reducción de los gastos en salud por medio de una atención basada en el principio de responsabilidad individual. El resultado de ese proceso permite fundamentar la creación de un "sistema de seguridad sanitaria mínima” en el que las personas se aseguran siguiendo un principio de prudencia que permite tanto la contención del gasto como la garantía de una atención sanitaria básica.

PALABRAS CLAVE: Cuidado sanitario - justicia distributiva - responsabilidad personal.

\section{DWORKIN'S DISTRIBUTIVE THEORY AND THE RIGHT TO HEALTH CARE}

\begin{abstract}
This essay reviews the health distribution theory of the American philosopher Ronald Dworkin, which is developed under the premise of reducing the expenses about health through a service based on the principle of individual responsibility. The result of this process can support the creation of a "minimum level of health security system" in which people ensure themselves following a prudence principle that allows the restraint and the guarantee of a basic health care.
\end{abstract}

KEY WORDS: Health care - personal responsibility - distributive justice.

Fecha de recepción: 29 de noviembre de 2011.

Fecha de aprobación: 23 de abril de 2012.

Este artículo forma parte del proyecto de investigación No 1120022 financiado por el Fondo Nacional de Desarrollo Científico y Tecnológico (FONDECYT), titulado "Una propuesta teórica para resolver los conflictos de equidad vigentes en el sistema privado de salud chileno".

** Doctora en Derecho. Académica de la Universidad de Valparaíso, Chile y de la Universidad Diego Portales, Santiago, Chile. Correo electrónico: alejandra.zuniga@uv.cl 
Sumario: 1) Introducción. 2) La distribución de la subasta y el seguro 3) Del principio del rescate al del seguro prudente. 4) ¿Puede Dworkin justificar el acceso universal a cuidado sanitario? 5) Conclusión. 6) Bibliografía.

\section{1) INTRODUCCIÓN}

La tesis de distribución sanitaria del filósofo estadounidense Ronald Dworkin se desarrolla bajo la premisa de la reducción de los gastos en salud por la vía de acordar una cobertura mínima universal de la mano del principio de responsabilidad individual. Dworkin rechaza aquella posición para la que la salud es un bien que, por su importancia, sería capaz de consumir todos los recursos de un Estado lo que, en condiciones de escasez, implica desconocer el costo real de nuestras elecciones. Por ello, se propone un modelo que recoge aquellas ventajas del mercado que obligan a la gente a un compromiso capaz de garantizar que, si el acceso al mercado es equitativo, entonces el resultado de la acción de las personas será considerado justo (justicia procesal).

El resultado de ese proceso, según veremos, será la creación de un 'sistema de seguridad sanitaria mínima' en el que son las personas las que se aseguran haciendo una evaluación cuidadosa del costo de sus elecciones. De este modo, el test de Dworkin presume que los propios individuos, al ser consultados sobre qué prefieren hacer con sus bienes y cuánto están dispuestos a gastar en salud, motivará reemplazar el 'principio de necesidad' por el de 'prudencia' al momento de decidir sobre la adjudicación correcta de los recursos sanitarios.

\section{2) LA DisTRIBUCión DE LA SUBASTA Y EL SEGURO}

La nueva izquierda, argumenta Dworkin, no rechaza el ideal de la igual consideración y respeto de las personas sino que refuta una de las interpretaciones que se da de ese ideal. Aquella que defiende que todos deben tener exactamente el mismo bienestar "de la cuna a la tumba, sin

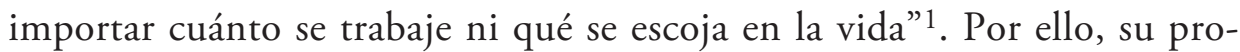
puesta llamada "igualdad de recursos" se desarrolla en torno a dos instituciones hipotéticas que permitirían acordar los términos de una cooperación social equitativa, respetando las diferencias entre las personas. Ellas son las instituciones de la subasta y el seguro.

Dworkin (2000) Sovereign Virtue. The Theory and Practice of Equality. Londres: Harvard University Press, p. 2. 
La igualdad de recursos busca, en concordancia con la teoría de la justicia del liberalismo igualitario al que pertenece, equiparar las circunstancias de las personas permitiendo que todos dispongan del mismo capital inicial (no del mismo nivel de satisfacción de preferencias) de forma tal que se les haga 'inmunes' a las influencias de circunstancias inmerecidas y, a la vez, 'responsables' de sus preferencias y ambiciones. La justa distribución de la propiedad solo se conseguiría cuando los recursos que controlan las diferentes personas sean iguales en sus "costes de oportunidad", es decir, en el valor que tendrían en manos de otras personas. Para evaluar esto el autor utiliza lo que llama test de la envidia que considera que la igualdad es perfecta cuando ningún miembro de esa comunidad envidia el conjunto total de recursos que está bajo el control de cualquier otro miembro.

La envidia, claro está, no es un concepto psicológico sino económico y permite decir que hay igualdad de recursos aun cuando la felicidad o el bienestar conseguidos por la gente mediante la igualación de sus bienes resultaran desiguales. "Si sus metas, ambiciones o proyectos son más fáciles de satisfacer que los míos, o si su personalidad es distinta en algún aspecto pertinente, ustedes pueden ser mucho más felices o estar más satisfechos con su vida de lo que yo lo estoy, a pesar de que yo no cambiaría mis recursos por los suyos. La igualdad liberal es igualdad de recursos, no de bienestar" 2 . Luego, idealmente es posible satisfacer esta prueba incluso aunque la distribución de las personas tenga niveles muy diferentes de prestaciones sociales o de bienestar ¿cómo? Imaginando un mecanismo artificial de repartición de bienes similar a la posición original de Rawls o al estado de naturaleza del contractualismo: "la isla desierta", donde una serie de personas se distribuyen los bienes que ahí encuentran apostando por diversos grupos de recursos físicos a partir de existencias iniciales equitativas representadas en, por ejemplo, conchas (que hace las veces de dinero). Esta 'subasta' se realiza repetidas veces en rondas sucesivas hasta que todo el mundo, al finalizar, está contento con su parte, es decir, la subasta acabará una vez que se satisfaga el 'test de la envidia' y nadie quiera el paquete de recursos que otro haya adquirido.

Este mecanismo, con todo, solo proporcionaría una distribución inicial que se verá alterada por todas las decisiones postsubasta que las partes tomen durante sus vidas con respecto al comercio, la producción y el consumo. Los recursos de los que disponga una persona en momentos distintos, así como el bienestar que le proporcionen, dependerán, no solo de sus propias decisiones, sino que también de las de los demás de modo que, para Dworkin, en tanto que las personas son diferentes no es necesario ni deseable que los recursos permanezcan siempre iguales pues quienes

2 Dworkin (1993) Ética privada e igualitarismo politico. Barcelona: Paidós, pp. 87-88. 
se han esforzado más y son más talentosos tiene derecho, siempre que no abusen de los demás, a obtener recursos extras.

Así, el modelo propuesto tiene por fin el diseño de instituciones políticas y económicas en pos de una igualdad de recursos lo más extensamente posible. Si bien es evidente que ninguna sociedad compleja y orgánica real daría lugar, a lo largo de la historia, a nada remotamente comparable a una subasta equitativa, el modelo sirve para preguntarse, en relación con una distribución actual, si ella cae dentro de la clase de distribuciones que se habrían producido en una subasta como esta, a partir de una descripción defendible de los recursos iniciales. En otras palabras, el mecanismo de la subasta puede proporcionar un criterio para juzgar hasta qué punto una distribución real, con independencia de cómo se haya logrado, se aproxima a la igualdad de recursos en un momento dado.

El segundo momento en el ejercicio imaginario de Dworkin opera una vez finalizada la subasta y tiene por objeto que a cada persona se le asigne una porción adicional de recursos para la adquisición de bienes con el fin de que sean utilizados para dos objetivos principales: 1) contratar seguros para hacer frente a eventuales desventajas futuras surgidas, fundamentalmente, por las diferentes capacidades con las que las personas nacen dotadas y 2) llevar a cabo el plan de vida que cada cual ha elegido. Ahora, sea como sea la distribución que se haga, los recursos controlados por la gente serán siempre distintos pues, si bien es posible repartir recursos impersonales (cosas susceptibles de apropiación y distribución, como la tierra, materias primas, bienes, etc.) los recursos personales (constituidos por cualidades de la mente y del cuerpo que influyen en el éxito de las personas, como la salud física y mental, la fortaleza y los talentos) no pueden transferirse.

Como es una subasta solo de bienes impersonales, los recursos personales seguirán siendo distintos una vez finalizada, de modo que el test de la envidia no podrá ser superado. Si se deja que los inmigrantes produzcan y comercien a su antojo la prueba de la envidia pronto fallará pues algunos serán más hábiles para producir lo que quieren o para comerciar con otros o simplemente trabajarán más y mejor. También puede ocurrir que haya personas que estén sanas mientras otras se enfermen o que algunos tengan suerte en los negocios y otros una muy mala fortuna. Por estas y otras tantas razones la igualdad pronto se frustrará y las personas preferirán el paquete del vecino al suyo. La igualdad liberal, entonces, deberá utilizar alguna estrategia compensatoria que repare, hasta donde sea posible, las desigualdades de recursos personales y de suerte y para ello se propone el 'mercado hipotético de seguros'.

Para comprender el tipo de seguro propuesto por Dworkin, se debe distinguir entre dos tipos de suerte, la suerte opcional y la suerte bruta. La suerte opcional depende de la aceptación de un riesgo aislado, previamen- 
te calculado, donde es posible ganar o perder. Si compro valores de la bolsa en alza, entonces mi suerte opcional es buena y, como ese riesgo lo asumo libremente y depende enteramente de mi voluntad, la compensación no es debida. La segunda clase, la suerte bruta, deriva de riesgos que la persona 'no ha escogido tomar' (por ejemplo, nacer ciego o sufrir un accidente sin culpa) por lo que la redistribución de recursos si sería debida. La diferencia entre ambos tipos de suerte es gradual y no siempre se puede tener certeza sobre su origen. Por ello, la institución del seguro tiene por objeto proporcionar una conexión entre ambos tipos de suerte de modo que la decisión de comprar o rechazar un seguro contra una catástrofe sea una apuesta calculada que transforme y 'convierta la suerte bruta en suerte opcional'. Sin bien el seguro no elimina la distinción original, en la medida en que se da la posibilidad de elegir o no un determinado seguro los hechos de suerte bruta se ubicarán en el campo de la suerte de opción.

Así, el seguro constituye un puente entre la suerte bruta y la suerte opcional donde lo que se intenta es trasformar los acontecimientos que podríamos calificar como suerte bruta en situaciones que califican dentro del campo de la suerte opcional. Si a X e Y se les ofrece un seguro contra la contingencia de quedar ciegos pero solo $\mathrm{X}$ toma el seguro y ambos sufren el accidente que les quita la vista, la tesis de Dworkin sostendrá que, si bien los dos tuvieron una mala suerte bruta "solo uno de ellos la convirtió también en mala suerte opcional al elegir no tomar el seguro"3. Por esto, la idea de la igualdad de recursos, al margen de cualquier ańadido paternalista, no defenderá que se redistribuyan bienes de la persona que se ha hecho un seguro para dárselos a quien no quiso tomarlo en el caso de que ambas quedaran ciegas en el mismo accidente, pues el hecho de disponer de un seguro significaría que, aunque ambas hayan tenido mala suerte bruta, la verdadera diferencia entre las dos ha sido una cuestión de suerte opcional. Luego, si se satisfacen las condiciones de equidad de Dworkin (que todos tengan el mismo riesgo de sufrir una catástrofe que los dejara discapacitados, que todos conocieran más o menos las probabilidades que tienen de que ello ocurra y que todos tengan la posibilidad de hacerse un seguro) las discapacidades no plantearían ningún problema a la igualdad de recursos.

Para Will Kymlicka la propuesta de Dworkin resulta deficiente pues en el mundo real el test de la envidia fracasará si las diferencias de las personas ya no tienen su origen en la elección sino que en la naturaleza. Quienes son discapacitados tienen más necesidades que los sanos de manera que no sería justo darles la misma cantidad de bienes para comprar

3 DwORKIN (2000) 76. 
en la subasta ${ }^{4}$. A este problema Dworkin responde sosteniendo que las personas discapacitadas (que requieren recursos extras para alcanzar una buena vida) deberán recibir, antes que se inicie la subasta, más recursos para compensar sus desventajas naturales no elegidas. Una vez que eso se ha hecho, damos a cada persona la misma cantidad de recursos y la subasta comienza igual para todos, de manera que cada cual elige los bienes que quiere conforme a su plan de vida. Así, compensando a las personas antes de la subasta se logra que el proceso sea 'sensible a las circunstancias' y después de haber equiparado las distintas capacidades de la gente, se entrega a todos la misma cantidad de recursos (dentro de la subasta) para que cada cual elija los bienes que quiera conforme a sus intereses con lo que, entonces, se logra que el proceso sea también 'sensible a las ambiciones'.

Esta respuesta, con todo, no funcionaría para todas las discapacidades. Según Kymlicka, no responde a aquellas discapacidades que generan desventajas durante toda la vida y que requerirían, para alcanzar la igualdad, una compensación constante. Por ejemplo, alguien con un severo retraso mental requerirá un gasto tal de bienes que no dejarían nada para que los demás alcanzaran sus propios proyectos de vida. Si los recursos se utilizaran en casos como estos para equiparar las circunstancias antes de que la subasta empiece, no quedaría nada para permitir que las personas actuaran siguiendo sus elecciones y ofertando recursos en la subasta ${ }^{5}$.

Una forma de realizar en la práctica la subasta hipotética sería a través del sistema de impuestos, que funcionara de manera similar a la subasta, cobrando a cada uno una cantidad suficiente para cubrir los gastos por diferencias de riquezas, de salud o desempleo que tengan su origen en la mala suerte natural. En este esquema sería irracional no asegurarse a nada y también lo sería asegurarse contra todo de modo que la cantidad de recursos que una sociedad gaste en protegerse contra las posibles calamidades será, para Dworkin, la cantidad justa. Este sistema impositivo, sin embargo, solo podría aproximarse a los resultados de la subasta. Lo anterior se debe, explica Kymlicka, a que no existe forma en el mundo real para medir que parte de las características de las personas son desventajas naturales y cuáles no lo son. Una razón es que las elecciones de las personas influyen en el desarrollo de sus talentos y las diferencias que tengan ese origen no merecerían compensación pues son fruto de la elección personal. Luego, resulta extremadamente difícil saber si las diferencias

Kymlicka, Will (2002) Contemporary Political Philosophy, an Introduction. 2a Edición. Londres: Oxford University Press, p. 77.

$5 \quad$ Si no podemos alcanzar la total igualdad ¿qué podemos hacer? Esta gran dificultad hace que tenga sentido que Rawls rehusara, en su Teoría de la Justicia, compensar las desventajas naturales y no las incluyera en el índice que determina a los que debemos considerar como los 'peor situados'. 
entre las personas se deben a su naturaleza distinta o a sus distintas elecciones. ¿Cómo decidir a quién compensar y a quien gravar con impuestos? La respuesta de Dworkin proponer gravar a los ricos -aunque lo sean solo fruto de su esfuerzo- y compensar a los pobres -aunque lo sean solo fruto de sus malas elecciones-6 ${ }^{6}$. Ello probablemente se debe a que recoge la idea fundamental de la Teoría de la Justicia de John Rawls relativa al carácter arbitrario de las dotaciones innatas y sociales de las personas y al modo estrecho en que unas influyen y determinan el desarrollo de las otras y, finalmente, los principio de justicia que han de regular las instituciones básicas de la sociedad ${ }^{7}$.

\section{3) DEL PRINCIPIO DEL RESCATE AL DEL SEGURO PRUDENTE}

Dworkin inicia su análisis sobre distribución de recursos sanitarios recordando que en su país, los Estados Unidos, existen más de 45 millones de personas que, o no tienen prácticamente ningún seguro médico o que lo perderán a corto plazo debido a que quedarán sin trabajo o contraerán alguna enfermedad o condición que los hará 'no asegurables'. Las preguntas cruciales ante este problema son, a su entender ¿cuánto debe gastar un Estado en salud? ¿Cuál es el nivel de atención médica que una sociedad decente debe ofrecer a todos? Y, como no podemos entregar a todo el mundo el nivel de atención que los ricos pueden comprar entonces ¿de qué modo decidiremos cuál es el nivel mínimo de atención sanitaria que es justo que todos reciban, incluidos los más pobres?

Cualquier racionalización del gasto en salud dependerá de una concepción acerca de qué tratamientos resultaría injusto negar a causa de sus elevados costes. Por ello el autor cuestiona la aplicación del principio del rescate que sostiene, por una parte, que el cuidado sanitario y la vida son los mayores y más importantes bienes y que todo lo demás debe ser sacrificado en su nombre y, por otra parte, que la atención de salud debe ser distribuida equitativamente. De este modo el principio del rescate responde a las dos preguntas cruciales -cuánto gastar y cómo distribuir el gasto- de la siguiente manera: sobre lo primero, el principio responde 'hay que gastar todo lo que se tenga' y sobre lo segundo, que 'el gasto debe distribuirse equitativamente'.

Este principio es tan antiguo y está tan extendido, sostiene Dworkin, que no se cuestiona que su uso, muchas veces, ha hecho más daño que bien pues no tiene sentido gastar todo en cuidado sanitario si no se gana, a cambio, prácticamente nada en expectativa de vida. Evidentemente nin-

Kymlicka (2002) 80.

Rawls, John (1971) A Theory of Justice. Oxford: Oxford University Press. 
guna sociedad sana puede hacer eso y ninguna persona individual organizaría su vida en torno a ese principio por lo que, concluye, es necesario rechazarlo por imposible. En lo que sí el principio del rescate ayuda es en el otro aspecto relacionado con la justicia: cómo hacer la distribución. Sobre esto propone descartar una distribución en base a la capacidad económica de las personas y prefiere utilizar el criterio de la necesidad. ¿Pero cómo se mide la necesidad sanitaria? Cuando alguien requiere de una operación para salvar su vida pero que tiene pocas probabilidades de éxito ¿es eso una necesidad? ¿Depende la necesidad de la calidad de vida que se espere se obtenga con el tratamiento? ${ }^{8}$.

Como alternativa al principio del rescate Dworkin propone el principio del seguro prudente que, para determinar el 'mínimo sanitario', ofrece el siguiente análisis teórico. Imaginemos una sociedad en la que se dan las siguientes tres características. Primero, el sistema económico provee de una distribución de los recursos basada en la 'justa igualdad', es decir, de una estructura económica que trata a todos los miembros de la comunidad con igual consideración, dividiendo los recursos en partes iguales y medidos de acuerdo con los costos de oportunidad que cada cual dé a un bien particular, dejando a cada persona en libertad para gastar sus recursos de la manera más apropiada y conforme con su plan de vida libremente diseñado9 9 . Segundo, la información sobre el costo, efectos secundarios y utilidad de los tratamientos, procedimientos y medicamentos -en otras palabras, todo lo que saben los doctores- estaría al alcance del público en general de modo que todos podrían acceder a ese conocimiento. Finalmente, y en tercer lugar, nadie en esa sociedad, incluyendo las compañías de seguros, poseería la información sobre los antecedentes y probabilidades que cualquiera pueda tener de contraer alguna enfermedad o sufrir algún accidente de manera que no pueda discriminarse a nadie por esas razones ${ }^{10}$. En estas condiciones ¿qué tipo de acuerdo sanitario se desarro-

$8 \quad$ El análisis costo-beneficio que sugiere Dworkin no es desconocido por los sistemas de salud públicos de países como Inglaterra donde los médicos, obligados a tomar decisiones difíciles, como la distribución de tratamientos de diálisis renal o de órganos para trasplantes, han construido guías médicas informales en las que se recomienda tener en cuenta la edad del paciente, su estado general de salud, la calidad de vida y sus expectativas, incluyendo la condición social y la posibilidad de recibir por amigos y familia los cuidados necesarios. Véase ZúñIga Fajuri, A. y De Lora, Pablo (2009) El derecho a la asistencia sanitaria. Un análisis desde las teorias de la justicia distributiva. Madrid: IUSTEL.

9 Dworkin (1993). "Justice in the Distribution of Health Care". McGill Law Journal, Vol. 38, No 4.

10 Sobre esto último Dworkin deja pendiente la cuestión de si es correcto, en el ejercicio hipotético construido, excluir información sobre los riesgos de una enfermedad causada por un comportamiento voluntariamente elegido. Por ejemplo ¿las compañías de seguros deberían cobrar más a los fumadores y a los escaladores de montaña? Esto parece razonable pero, si lo es ¿qué es lo que cuenta como un comportamiento voluntario? Por ejemplo ¿’lo es un comportamiento sexual determinado?, ¿sería así porque las personas tienen menos control sobre sus conductas 
llaría? ¿Cuánto de los recursos de esa comunidad se destinarán a salud y cómo se distribuirían entre sus miembros?

El modelo de Dworkin supone asignar recursos para salud y otras necesidades sociales a los pacientes que requieren tratamiento, intentando imaginar cómo sería la atención sanitaria si se dejara en manos de un mercado libre no subsidiado que, sin embargo, pudiera corregir las tres deficiencias mencionadas. De este modo habría que suponer que en esta comunidad las decisiones sobre salud se toman como decisiones individuales, en un mercado libre donde compañías de seguros y médicos pueden cobrar por sus prestaciones tanto como quieran. Así, el autor construye una especie de posición original en la que, al no haber inequidades sociales, las personas pueden elegir el paquete de 'seguro sanitario' que desean comprar con los bienes que poseen y teniendo presente el costo de oportunidad que esos recursos sanitarios les signifiquen.

De este modo el procedimiento concluirá que, en primer lugar, sea lo que sea que esa comunidad gaste en salud en su conjunto, esa será la cantidad justa; en segundo lugar, el modelo de distribución de la atención médica en esa sociedad será justo solo para esa sociedad. Luego, la hipótesis será: una distribución justa en salud es aquella que una persona bien informada crea para sí misma mediante su elección individual suponiendo que el sistema económico y la distribución de la riqueza en esa comunidad son justas. "Esta importante conclusión nos ayudará a decidir qué tipo de atención de salud deberíamos proveer a todos en nuestra comunidad imperfecta e injusta. Podemos especular sobre qué clase de seguro la gente se haría y luego usar los resultados de esa especulación como guías para decidir qué requiere la justicia en este tema"11.

Las reglas que seguramente se seleccionarían serán: primero, la obligatoriedad de racionalizar recursos sanitarios; segundo, que lo que cada cual decida gastar dependerá de sus planes de vida autónomos y; tercero, si bien no podemos saber que elegirá cada cual sí podemos formarnos algún juicio sobre algunas necesidades y preferencias generales de las personas. Por ejemplo, para la mayoría de las personas jóvenes sería irracional costear un seguro carísimo que les cubriera en caso de ingresar en un estado vegetativo persistente, pues los costos de oportunidad serían demasiado altos. Siempre será mejor gastar bien ese dinero durante el tiempo que se tenga antes de caer en esos estados, es decir, seguramente la mayoría preferirá invertir ese dinero en mejorar la vida consciente. Este criterio, continúa, podemos extenderlo a los casos de demencia senil, de Alzheimer y otros estados de demencia o de inconsciencia. La gente contrataría,

sexuales que sobre su adicción a la nicotina o porque el sacrificio de renunciar al sexo es mayor que el dejar de fumar? Dworkin (2000) 311-312. 
posiblemente, un cuidado en condiciones dignas para esos casos, pero no tratamientos muy costosos que limiten sus planes de vida.

Con esa idea en mente podemos imaginar que la mayoría de las personas prudentes con recursos medios suficientes estaría dispuesta a comprar un seguro que cubriera un tratamiento médico 'ordinario' (hospitalización, atención prenatal, pediátrica, exámenes regulares y otro tipo de medicina preventiva). En esta sociedad todos tendrían ese mínimo y, si bien habría personas que, excepcionalmente, quisieran asegurarse a todo evento sin importar el coste, no parece justo exigir que todos los demás hicieran lo mismo a través de un sistema obligatorio. Luego, diferencias más o menos entre los gustos personales, al autor le parece equitativo elaborar un esquema de seguro obligatorio que se construya en función de lo que la mayoría, y no la excepción de un pequeño grupo de personas, juzga apropiado, siempre que se permita a esos pocos, que posean el dinero para ello, contratar un seguro complementario. En este sentido, Dworkin considera que el sistema sanitario adecuado es el sistema pluralista, es decir, un sistema sanitario estatal que permita la existencia de sistemas de seguros privados que puedan entregar servicios 'adicionales' más caros a las personas que puedan pagarlos ${ }^{12}$.

\section{4) ¿PUEDE DWORKIN JUSTIFICAR EL ACCESO UNIVERSAL A CUIDADO SANITARIO?}

La creencia de que una sociedad justa debe perseguir una política de acceso al cuidado sanitario universal es una creencia poderosa. Este acceso universal existe cuando a todos los ciudadanos, independientemente de su clase social, raza o género, se les garantiza la disposición de un conjunto de productos, servicios sanitarios básicos y medicamentos necesarios. Lesley Jacobs examina si la teoría liberal igualitaria de Ronald Dworkin puede justificar el acceso universal al cuidado sanitario sobre la base de las que serían sus dos demandas principales: primero, la afirmación de que, más allá de las diferencias que existen entre las teorías contemporáneas de la justicia, existe un acuerdo básico y fundamental en todas ellas sobre lo que llama el 'principio igualitario abstracto' que dice que los gobiernos deben actuar de forma que hagan que las vidas de aquellos a quienes gobiernan sean mejores vidas y de forma que muestren igual consideración respecto de cada una de ellas. El segundo principio sostiene que, para lograr ese trato igualitario, los gobiernos deben diseñar mecanismos de distribución equitativa de los recursos privadamente poseídos de modo

\footnotetext{
12 Para una clasificación de los Sistemas Sanitarios, véase ZúÑIgA, F. A (2007). "Sistemas sanitarios y reforma AUGE en Chile”. Revista Acta Bioethica. Año XIII, N².
} 
que se logre mostrar una igual consideración sensible a las diferentes ambiciones, metas y decisiones de cada individuo. Jacobs defiende que tanto la interpretación de Dworkin del principio igualitario abstracto como su requisito 'sensible a la ambición' son incompatibles con una política de acceso universal al cuidado sanitario ${ }^{13}$.

En el corazón de la teoría distributiva de Dworkin destaca su distinción entre la personalidad individual y las circunstancias. En la primera el autor incluye cosas como las convicciones, las ambiciones, los gustos y las preferencias. En la segunda, los recursos, talentos, habilidades y capacidades tanto físicas como mentales. Ahora, la teoría de igualdad de recursos de Dworkin sostiene que las personas son tratadas como iguales cuando su parte en los recursos sociales durante toda su vida son iguales considerando un esquema distributivo sensible al costo. Ese costo se mide determinando cuán valiosos son para los demás los recursos y elementos que una persona consume, lo que Dworkin llama el 'costo de oportunidad' de la propia vida. ¿Cómo una tesis igualitaria debe responder a la realidad de las circunstancias injustas originadas en la enfermedad o discapacidad, es decir, en las diferencias 'inmerecidas' en salud? ¿Permite su igualdad de recursos originaria asegurar el acceso universal a cuidado sanitario? Para Jacobs la respuesta es 'no' pues el punto central de esta discusión se basa en que una política de acceso universal al cuidado sanitario envuelve, necesariamente, el uso de transferencias de especies (servicios sanitarios) de modo que no se permita que los individuos cambien esos bienes por dinero o por bienes de distinta naturaleza, como educación, comida, vivienda, diversión etc., como defiende, en cambio, el mecanismo de 'seguro prudente' de Dworkin. Esto quiere decir que no es posible reemplazar la 'transferencias de bienes' por 'transferencia de dinero' si se quiere asegurar el acceso sanitario universal. Y es que, si bien a veces el tipo de transferencia o redistribución que se utilice para igualar a las personas puede variar de un tipo a otro sin mayores problemas, en ciertas circunstancias "las justificaciones normativas para una política distributiva entrañan el uso específico de trasferencias de dinero o de transferencias en especies"14.

¿Puede el mercado hipotético de seguros justificar el uso de transferencias redistributivas en especies? Jacobs cree que no pues una distribución justa, según Dworkin, debe ser sensible a la ambición lo que naturalmente lleva a la necesidad de organizar el sistema en torno al mercado y al dinero, que permiten que cada persona decida qué bienes comprar ya que 'el dinero es inherentemente sensible a la ambición'. Este problema puede apreciarse mejor a través de un ejemplo: Imaginemos a una perso-

13 Jасовs, Lesley A. (2004) Dworkin and his Critics: with Replies by Dworkin. Oxford: Editor Justine Burley. Blackwell Publishing, p. 134.

14 JaCOBs (2004) 140-141. 
na que ha contraído, debido a su mala suerte, una grave enfermedad que, sin embargo, puede tratarse por medio de una operación. Supongamos también que esta persona es ciudadano de una sociedad que garantiza el acceso universal al sistema sanitario y que la operación en cuestión está cubierta por el sistema. El sujeto enfermo, con todo, es especial pues solicita al Estado que, en vez de asegurarle la operación, le entregue el dinero equivalente para comprar un bien que considera más valioso. En el sistema 'sensible a la ambición' de Dworkin ¿qué razón habría para negárselo? ¿Qué habría de malo en que el sistema compensara la falta de salud de las personas con dinero que ellas podrían gastar en bienes que valoran más?

Una transferencia de especies tiene por objeto igualar a las personas en ciertos bienes particulares, lo que supone un criterio objetivo para definir una distribución igual de los recursos sociales pues se estima que son esos recursos y no otros los más importantes, los que se deben 'igualar', sin considerar el orden relativo que el receptor de esos recursos pueda haber hecho. Si bien el propio Dworkin admitiría este 'paternalismo superficial' -que obligaría a las personas a tomar precauciones razonables dentro de su propia estructura de preferencias- Jacobs considera que, dentro del esquema de distribución de la igualdad de recursos 'sensible a la ambición', muchas veces ese paternalismo no podría justificarse, como en el caso de personas con enfermedades incurables, por lo que la teoría de Dworkin no podría defender el acceso universal a cuidado sanitario.

A esta crítica Dworkin responde que, debido a que la gente puede tomar malas decisiones sanitarias, sería irresponsable permitirles no tomar seguros. Además, argumenta, existe otra razón para admitir un cierto grado de paternalismo en este sentido: las externalidades. Ello pues los ciudadanos enfermos son tan caros para la economía social como para los empleadores privados $y$, además, resultan particularmente caros para una comunidad decente que no querría permitir que murieran en la indigencia o por falta de cuidado médico, aun cuando hubieran gastado todos sus recursos en otras cosas. Por esto, concluye, un gobierno comprometido con los principios generales de la igualdad de recursos deberá limitar la libre elección en ciertas circunstancias de modo que "El esquema de cuidado sanitario que defiendo en Sovereign Virtue no permite que los beneficiarios sustituyan los tratamientos por dinero" 15 .

Finalmente ¿responde el sistema sanitario ideado por Dworkin a todos los dilemas que genera el hacer a las personas 'responsables por su estatus sanitario'? El autor considera errada la opinión de que la igualdad de recursos es un mecanismo implacable que declara que si las personas hacen malas elecciones y arruinan sus vidas la comunidad no debe ayu-

15 Dworkin (2004) Dworkin and his Critics: with Replies by Dworkin. Oxford: Editor Justine Burley. Blackwell Publishing, pp. 360-361. 
darles pues ello invadiría injustamente los recursos de otros que no han cometido los mismos errores. En este sentido, rechaza la visión de autores como Arneson o Fleurbaey que han sostenido que la teoría resulta idéntica a la tesis del "punto de partida" pues implica que las personas comienzan con los mismos recursos y que de ahí en adelante se hacen cargo solos de las consecuencias de sus propias decisiones. Al contrario, Dworkin argumenta que sus críticos han omitido o desatendido el mecanismo del seguro hipotético pues la igualdad de recursos protege a los individuos de decisiones económicas desastrosas a través de un esquema de impuestos y provisiones de bienestar que es modelado por el seguro que la teoría supone que toda persona 'normal' de la comunidad, actuando prudentemente, adoptaría. Si se asume que ese esquema proveería a todos con, al menos, un estándar de vida mínimamente decente, entonces no habrá nadie que llegue a estar en una situación de desamparo a consecuencia de sus malas decisiones, como la imaginada por los críticos.

¿Debiera la igualdad de recursos insistir en que quien ha sido gravemente dańado, pero no ha tomado el seguro y no puede costearse el tratamiento médico, debe ser abandonado hasta morir desangrado en la calle? No. En esa circunstancia una comunidad comprometida con la igualdad de recursos tiene suficiente justificación para exigir que las personas se aseguren para, al menos, un cuidado médico básico, como en los hechos la mayoría de las comunidades actuales lo hacen. Un seguro básico obligatorio estaría, entonces, justificado por dos razones: la primera apela al 'principio de corrección' por el cual, si alguien falla en asegurarse contra los accidentes personales y además carece de medios para costearlos, la comunidad se hará cargo. "La igualdad de recursos necesita un principio de corrección para ese tipo de externalidades, y ese principio permite los seguros obligatorios". La segunda razón, prosigue Dworkin, es abiertamente paternalista: una sociedad decente debe proteger a las personas contra los grandes errores que seguramente lamentarían, como no haber usado el cinturón de seguridad o no haber comprado un seguro contra las emergencias médicas ${ }^{16}$.

Una última crítica a la propuesta dworkiniana que vale la pena destacar concuerda en que las 'injusticias' mencionadas se dan toda vez que se niega a quien lo necesita los medios mínimos de atención sanitaria en razón de su supuesta 'irresponsabilidad'. Y es que parece prácticamente imposible identificar con claridad, para ser justos, aquellos factores de los que realmente se es individualmente responsable. En este sentido, Beauchamp y Childress se preguntan si, en el caso de la gente que lleva a cabo voluntariamente acciones que significan una pérdida de salud (por ejemplo, quienes han adquirido Sida a consecuencia de actividades sexuales 
poco seguras o por uso de drogas por vía intravenosa, los fumadores con cáncer al pulmón y los alcohólicos que desarrollan una enfermedad del hígado) ¿tiene la sociedad la misma obligación de proporcionarles atención sanitaria como lo hace con pacientes que necesitan atención a causa de la mala suerte?

Una política de negación de los fondos sociales no puede, según los autores, justificarse a menos que se den varias condiciones. Primero, debe ser posible identificar y diferenciar los factores causales en la morbosidad, tales como causas naturales, ambiente social y actividades personales. "Entonces debe confirmarse que una enfermedad es inevitablemente resultado de las actividades personales, más que de alguna otra causa". Debe también demostrarse que las actividades personales en cuestión eran autónomas, en el sentido de que los actores eran conscientes de los riesgos y los aceptaban. El problema de la primera condición es que es virtualmente ilusorio aislar los factores causales para muchos casos críticos de mala salud, en razón de la complejidad de las conexiones y límites del conocimiento. "Las necesidades médicas son frecuentemente resultado de la conjunción de predisposiciones genéticas, acciones personales y condiciones ambientales y sociales. Es a menudo imposible establecer los papeles respectivos de estos diferentes factores, con razonable certeza, sobre la base de pruebas científicas" 17 .

Además de estos obstáculos, habría que agregar los problemas de vigilancia de los sistemas sanitarios pues, para determinar de modo preciso las condiciones causales de las cuestiones concretas de salud y para localizar a quienes se arriesgan voluntariamente, los oficiales investigadores tendrían que estar autorizados a invadir la intimidad, romper la confidencialidad y realizar minuciosos registros para documentar los abusos de salud que pudieran dar como resultado una pérdida del derecho a un tipo particular de asistencia sanitaria. Tal aplicación sería costosa y, además, de rasgos moralmente desagradables ${ }^{18}$. Por último, si bien una razón mayor para discutir sobre la pérdida de los derechos a la atención sanitaria es el incremento de los costes, a veces la prevención de riegos a través de alteraciones en el estilo de vida y la conducta podrían llevar a resultados imprevistos. En efecto, algunas actividades arriesgadas requieren, en verdad, menos que más atención sanitaria, porque el resultado de ellas son muertes más tempranas y rápidas que las que podrían ocurrir si el indi-

17 Beauchamp y Childress (2009) Principles of Biomedical Ethics. 6a edición. New York: Oxford University Press.

18 ¿Cómo distinguir qué desventajas son voluntarias y cuáles involuntarias? Intentar semejante distinción obligaría al Estado a ver a los ciudadanos con desconfianza y como potenciales estafadores del sistema de compensaciones de modo que, con el objeto de evitar esa desconfianza, los desaventajados se comprometerían con lo que Wolff llama la "revelación de la vergüenza", teniendo que probar que sus desventajas sí son involuntarias, debido a sus talentos naturales o a las características de su infancia. KymLicka (2002) 198. 
viduo viviera más tiempo y desarrollase un proceso debilitante crónico y largo.

\section{5) CONCLUSIÓN}

Hoy más que nunca se precisa insistir en el análisis de aquellas teorías de la justicia que han pretendido fundamentar, moralmente, el acceso a un cuidado sanitario básico universal, junto con algún mecanismo de contención de costes sanitarios. No podemos olvidar que las sociedades democráticas modernas obligan a admitir que no es posible garantizar el principio de igualdad equitativa de oportunidades -fundamento moral de la adjudicación de recursos escasos en una sociedad justa- sin que esté asegurado el derecho a cuidado sanitario para todos sus ciudadanos, sin discriminación.

A su vez, los cambios epidemiológicos que están sufriendo las sociedades modernas, producto del envejecimiento de su población y del aumento en las expectativas de vida, hacen crucial considerar la distinción que Dworkin realiza en torno al principio de prudencia, abogando porque los ciudadanos se hagan cargo del costo de sus elecciones en materia de cuidado sanitario y apostando por generar un modelo que garantice un resultado justo si se cumple el procedimiento propuesto.

\section{6) BIBLIOGRAFÍA}

- Beauchamp y Childress (2009) Principles of Biomedical Ethics. 6a edición. New York: Oxford University Press.

- Dworkin, Ronald (1993a) Ética privada e igualitarismo político. Barcelona: Paidós.

- Dworkin, Ronald (1993b). "Justice in the Distribution of Health Care”. McGill Law Journal, Vol. 38, No 4.

- Dworkin, Ronald (2000) Sovereign Virtue. The Theory and Practice of Equality. Londres: Harvard University Press.

- Dworkin, Ronald (2004). Dworkin and his Critics: with Replies by Dworkin. Oxford: Editor Justine Burley. Blackwell Publishing.

- Jaсовs, Lesley A. (2004) Dworkin and his Critics: with Replies by Dworkin. Oxford: Editor Justine Burley. Blackwell Publishing.

- Kymlicka, Will (2002) Contemporary Political Philosophy, an Introduction. 2a Edición. Londres: Oxford University Press.

- Rawls, John (1971) A Theory of Justice. Oxford: Oxford University Press. 
- ZúÑiga F., Alejandra y De Lora, Pablo (2009) El derecho a la asistencia sanitaria. Un análisis desde las teorías de la justicia distributiva. Madrid: IUSTEL.

- ZÚNiga F., Alejandra (2007). "Sistemas sanitarios y reforma AUGE en Chile". Revista Acta Bioethica, Año XIII, Nº 2. 